\title{
N-Substituted Acrylamide-Styrene Copolymer Latices III. Morphology of Latex Particles
}

\author{
Futoshi Hoshino, Haruma KawaguchI, and Yasuji OHtsuKa \\ Department of Applied Chemistry, Faculty of Science \& Technology, Keio University, \\ 3-14-1 Hiyoshi, Kohoku-ku, Yokohama 223, Japan
}

(Received December 16, 1986)

\begin{abstract}
Styrene (St)- $N$-acryloyl pyrrolidine (APr) copolymer latices were prepared by soap-free emulsion copolymerizations and the morphology of the latex particles was investigated. The shape of particles significantly depended on the polymerization conditions and reflected their inner structures which were examined by the electron microscopy of $\mathrm{OsO}_{4}$-stained and thinly sectioned particles. The wide variation of inner structures from a mostly uniform one to an extremely phase-separated one arose from differences in the copolymerization modes of the two monomers and the diffusibility of propagating chains in growing particles depending on polymerization conditions.

KEY WORDS Soap-Free Emulsion Polymerization / Latex / Particle / Morphology / Phase Separation /
\end{abstract}

The morphology of latex particles affects the properties of latices themselves and films obtained from them. Therefore, it is very important to understand how to control the morphology of particles in order to prepare latices for specific purposes. Many studies have been done on the control of particle morphology in seeded emulsion polymerizations. ${ }^{1-11}$ According to these studies, most seeded emulsion polymerizations result in the formation of particles having heterogeneous inner structures such as a core-shell structure, POO (polymeric oil in oil) structure, or more complicated ones. The occurrence of such structures has been attributed to some phase separation inside the particles in most cases. ${ }^{711}$ Contrary to a large number of studies on seeded emulsion polymerizations, there have been only a few studies on the morphology of particles in usual emulsion copolymerization. ${ }^{12-14}$ In the typical soap-free emulsion copolymerization of a hydrophobic monomer with a hydrophilic monomer, the latter is apt to polymerize preferentially at the initial stage of polymerization to form hydrophilic polymerrich particle nuclei and the following polymerization proceeds in a manner similar to seeded emulsion polymerization. ${ }^{15,16}$ The soap-free emulsion copolymerization of styrene (St) with $N$-acryloyl pyrrolidine (APr) is one such example ${ }^{17}$ and resulted in the formation of latex particles having different shapes from a normal spherical one to an uneven-shaped one depending on the polymerization conditions.

In this paper, the relation between polymerization conditions and particle morphology was studied in St-APr copolymer system by means of electron microscopic observations and the polymerization mechanism is discussed.

\section{EXPERIMENTAL}

\section{Materials}

$\mathrm{APr}$ and $N$-acryloyl piperidine (APp) were offered by Mitsui Toatsu Chemicals Inc., and St was purchased from Wako Chemicals Co. These monomers were distilled under re- 
duced pressures $\left(89^{\circ} \mathrm{C} / 0.5 \mathrm{mmHg}, 85^{\circ} \mathrm{C} / 0.05\right.$ $\mathrm{mmHg}$, and $46^{\circ} \mathrm{C} / 21.5 \mathrm{mmHg}$, respectively). Acrylamide (AAm) was recrystallized from benzene. $N, N^{\prime}$-Azobis(2-amidinopropane) dihydrochloride (V-50, Wako Chemicals Co.) was used without further purification.

\section{Preparation of St-APr Latex (SAPr) Particles}

Soap-free emulsion copolymerization was carried out in a $300 \mathrm{ml}$ four-necked roundbottom flask equipped with stirrer, nitrogen inlet, condenser, and serum rubber cap in the temperature range from 40 to $70^{\circ} \mathrm{C}$. A standard recipe for the copolymerization is as follows: (St + APr) $30 \mathrm{~g}$, water $180 \mathrm{~g}$, and V-50 $0.5 \mathrm{~g}$. The weight fraction of APr in monomer feed (f) was varied from 0.1 to 0.4 . The conversion was determined gravimetrically. All the latices were purified by repetitive centrifugation-decantation-redispersion to remove water soluble polymers and electrolytes.

\section{Preparation of Poly(APr) Gel Particles}

Poly(APr) gel particles were prepared by the copolymerization of $\mathrm{APr}$ with $N, N^{\prime}$ methylene-bisacrylamide according to the method in the literature. ${ }^{18}$ The degree of swelling of poly(APr) gel particles was measured as follows: dry poly(APr) gel particles of weight $W_{\mathbf{d}}$ were swollen with solvents in a test tube. The sample was kept standing at room temperature overnight to complete the equilibrium swelling. The swollen particles were then filtrated and weighed. The degree of swelling was defined as $W_{\mathrm{w}} / W_{\mathrm{d}}$ where $W_{\mathrm{w}}$ was the weight of swollen gel particles.

\section{Electron Microscopic Observation}

The contour of particles was observed by a Hitachi HU-12AF transmission electron microscope and a FESEM-JFSM 30 scanning electron microscope.

The inner structure of the particles was studied as follows; latices of $5 \%$ solid were mixed with the same amount of $1 \% \mathrm{OsO}_{4}$ in a test tube, kept standing at a room temperature overnight, and freeze-dried in vacuo. The stained particles were poured into epoxy resins and cured at $60^{\circ} \mathrm{C}$ for 2 days. The cured samples were sliced into thin sections of $c a$. $60 \mathrm{~nm}$ thickness by using a LKB 4801A microtome and their transmission electron $\mathrm{mi}$ crographs were taken.

\section{RESULTS AND DISCUSSION}

\section{Effects of Polymerization Temperature and}

Monomer Composition on Morphology

Polymerizations were carried out in the temperature range from 40 to $70^{\circ} \mathrm{C}$. The polymerization at $40^{\circ} \mathrm{C}$ resulted in the formation of a very unstable latex. The latex prepared at $70^{\circ} \mathrm{C}$ had a very broad distribution of particle size. For these reasons, the effect of reaction temperature on particle morphology is discussed upon the latex particles prepared in the temperature range from $45^{\circ} \mathrm{C}$ to $60^{\circ} \mathrm{C}$. Their electron micrographs are shown in Figure 1. The figure also shows the dependence of particle shape on monomer composition in addition to its temperature dependence and indicates that the monomer composition is a more effective factor deciding particle shape. The influence of polymerization temperature on the inner structure of particles was examined by observing the transmission electron micrographs of $\mathrm{OsO}_{4}$-stained thin sections of SAPr particles in Figure 2. The dark domains represent APr-rich regions. The particles prepared at $50^{\circ} \mathrm{C}$ consist of three phases, that is, an APr-rich core, a St-rich shell, and an APr-rich skin. On the other hand, the particles prepared at $60^{\circ} \mathrm{C}$ had more complicated structure. They had no or only a little APr-rich core and uneven-shaped thick APrrich surface layer.

The difference in morphology between these two latex particles is attributed to the temperature-dependent hydrophilicity of poly(APr) and additionally to change of viscosity in growing particles. The polymerization of St with APr in a heterogeneous 


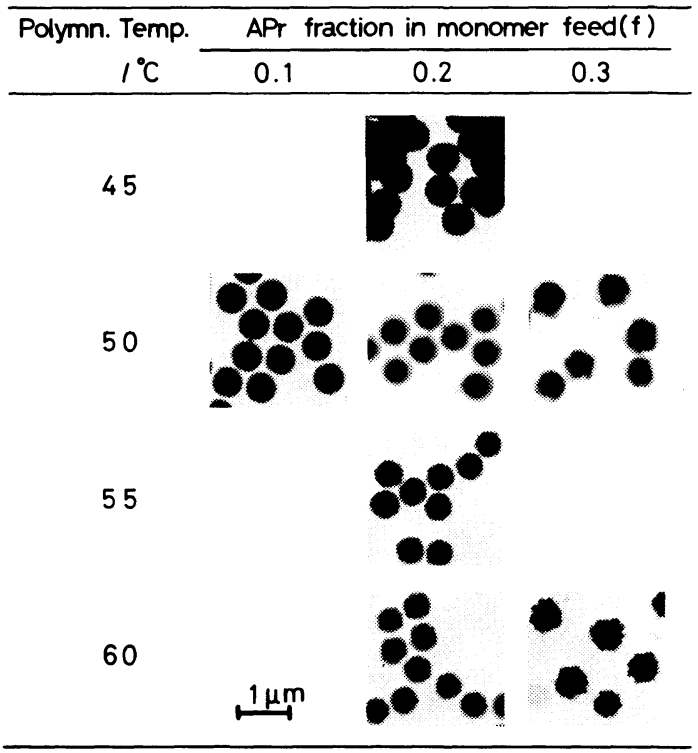

Figure 1. Transmission electron micrographs of StAPr latex particles prepared under different conditions. Recipe: monomer-water-V50 $=30: 180: 0.5(\mathrm{~g})$.

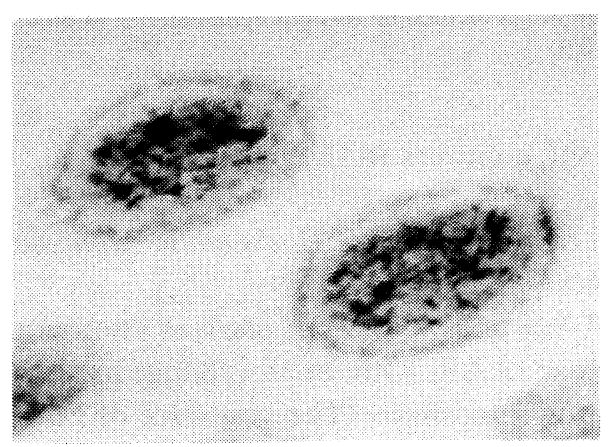

(A)

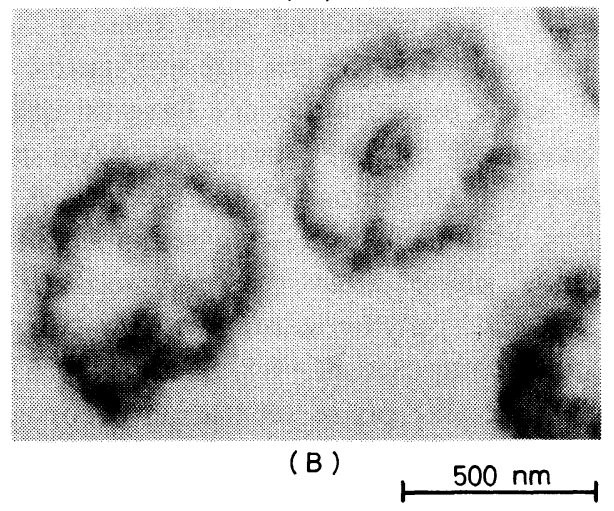

Figure 2. Transmission electron micrographs of $\mathrm{OsO}_{4}$ stained and thinly sectioned St-APr particles. Latex preparation: $f=0.2$; polymerization temperature $=(\mathrm{A})$ $50^{\circ} \mathrm{C}$ and (B) $60^{\circ} \mathrm{C}$.

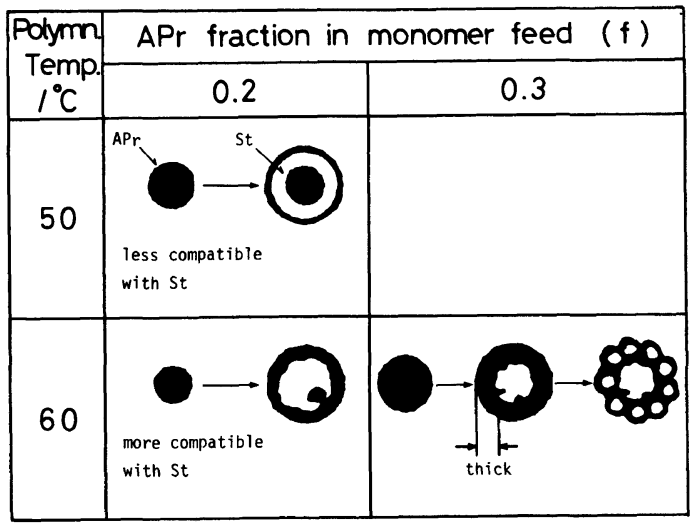

Figure 3. Schematic illustration of the effects of polymerization temperature and APr fraction in monomer feed $(f)$ on particle morphology.

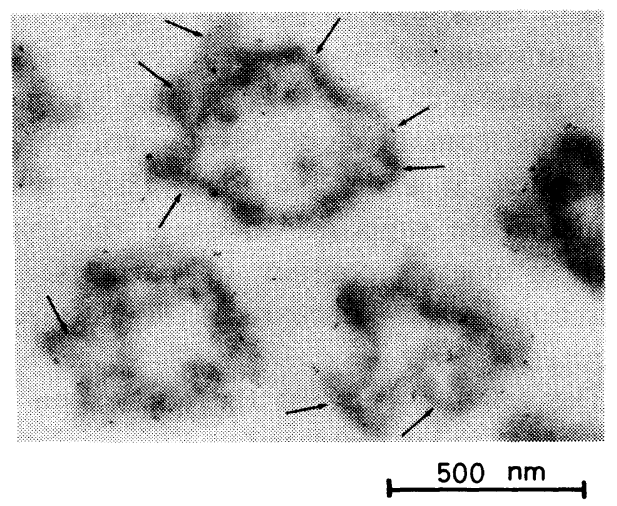

Figure 4. Transmission electron micrograph of $\mathrm{OsO}_{4}$ stained and thinly sectioned $\mathrm{St}-\mathrm{APr}$ particles. Latex preparation: $f=0.3$, temperature $=60^{\circ} \mathrm{C}$. Arrows indicate small St-domains existing just inside the projections of particles.

system can be regarded as a two stage or seeded polymerization due to the preferential polymerization of APr and delayed polymerization of St. ${ }^{19}$ The low compatibility of poly(APr) with poly(St) is supposed to result in the formation of phase-separated structure in each particle. Especially at a low temperature at which the difference in hydrophilicity between two polymers is large and the visocity in growing particles is high, it is probable that preferentially-polymerized APr seeds are hard to mix with or migrate through the $\operatorname{poly}(\mathrm{St})$ phase formed at a delayed time and they 
stay as cores in growing particles at $50^{\circ} \mathrm{C}$. On the contrary to this, poly(APr) formed at the initial stage in the polymerization at $60^{\circ} \mathrm{C}$ can mix partially with poly(St) and migrate through it due to its decreasing hydrophilicity and visocosity. A schematic illustration on temperature-dependent morphology is presented in Figure 3.

Figure 4 is an electron micrograph of the $\mathrm{OsO}_{4}$-stained thin section of SAPr particles prepared with $f=0.3$ at $60^{\circ} \mathrm{C}$. In each particle in the figure, there are some individual poly(St) micro-domains (white domains unstained by $\mathrm{OsO}_{4}$ which are indicated by arrows) buried in poly(APr) layer. A large fraction or thick layer of poly(APr) must restrict the diffusion of St-rich propagating chains and, consequently, give a number of microdomains composed of St-rich components that enter at a delayed time. These microdomains are supposed to cause the formation of uneven contour of particles. This situation is also schematically illustrated in Figure 3.

The relation between the conversion and particle shape is presented in Figures 5 and 6 for the polymerization with $f=0.3$ at $60^{\circ} \mathrm{C}$. According to Fugure 5, the polymerization was accelarated at about $50 \%$ conversion. This conversion corresponds to the one when all of St droplets have been exhausted and then the concentration of $\mathrm{St}$ in particles decreases with increasing conversion. As shown in Figure 6, particle shape started to be uneven at about the same conversion. This coincidence implies that the increase in the viscosity in particles is responsible for the formation of the uneven structure. Namely, newly entering radicals react with $\mathrm{St}$ monomer dissolving in poly(APr)-rich layer and lose their diffusibility due to the increasing viscosity in the particles. Propagation of unmovable radicals at multiple sites in each particle gives rise to the high rate of polymerization and the formation of a large number of poly $(\mathrm{St})$ domains in the particle. These domains give charac-

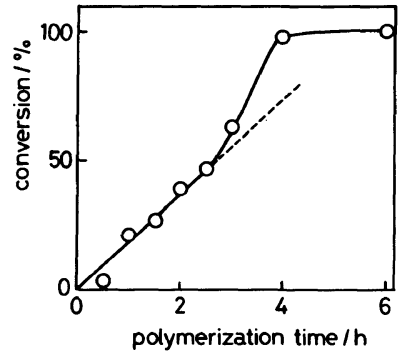

Figure 5. Time-conversion curve of soap-free emulsion copolymerization of St with $\operatorname{APr}$ at $60^{\circ} \mathrm{C}$ and $f=0.3$.

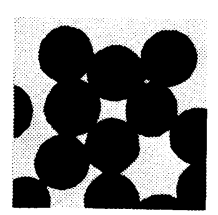

Conv. $\%: 46.3$

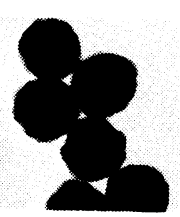

62.6

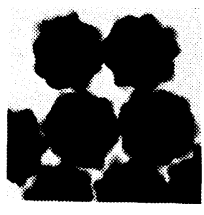

90.3

$500 \mathrm{~nm}$
Figure 6. Morphology change of St-APr particles with increasing conversion in the polymerization at $60^{\circ} \mathrm{C}$ and $f=0.3$.

teristic contours to the particles.

\section{Effect of the Kind of Hydrophilic Monomer on Morphology}

AAm and APp were used as comonomers instead of APr without any change in polymerization conditions. The contours of St-AAm and $\mathrm{St}-\mathrm{APp}$ particles are shown in Figure 7 and they are compared with that of SAPr particles which is the last one in Figure 6.

$\mathrm{St}-\mathrm{AAm}$ particles did not have an uneven surface such as SAPr particles. AAm is so hydrophilic that most of the charged AAm, that is, about $90 \%$ of AAm remained in the aqueous phase after the polymerization. ${ }^{15}$ As a result, polymerization with $f=0.3$ gave particles composed of $96 \%$ St and $4 \%$ AAm. No appreciable phase separation occurred in such St-AAm particles so that the particles were spherical having a smooth surface. The fraction of APr composing latex particles was determined to make clear the difference in the behavior between AAm and APr. The results 


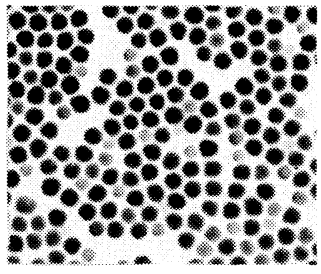

(A)

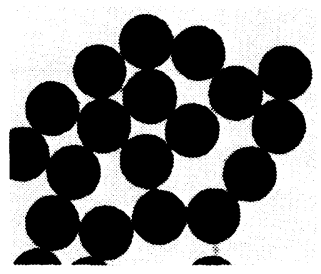

(B)
Figure 7. Transmission electron micrographs of (A) St-AAm and (B) St-APp latex particles.

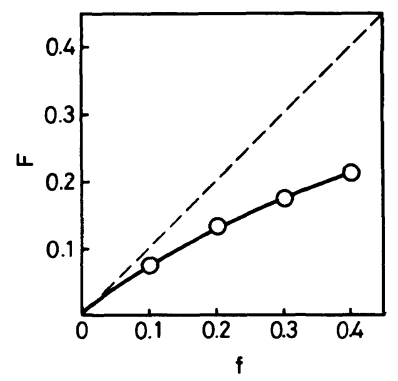

Figure 8. APr fraction in resulting polymer particle $(F)$ as a function of APr fraction in monomer feed $(f)$.

are shown in Figure 8. The existence of a relatively large amount of $\mathrm{APr}$ in each particle must restrict the diffusion of St-rich propagating chains and give uneven-shaped particles.

St-APp particles have also a smooth contour as do $\mathrm{S} \dot{\mathrm{t}}-\mathrm{AAm}$ particles. But the reason for the formation of such particles is different between St-AAm and St-APp systems. In the latter, charged A.Pp units mostly locate in or on the particles and the amount of APp units remaining in the aqueous medium is negligible due to low hydrophilicity. Furthermore, APp copolymerizes better with St than AAm or APr does because it has higher compatibility with $\mathrm{St}$ and suitable monomer reactivity ratios, $r_{1}=0.31$ and $r_{2}=2.17\left(M_{1}=\mathrm{APp}\right)(20)$, which partly cancels the overwhelming polymerization of APp caused by the difference in concentrations of $\mathrm{APp}$ and $\mathrm{St}$ in water. The high copolymerizability of St with APp prevents phase separation and results in the for-

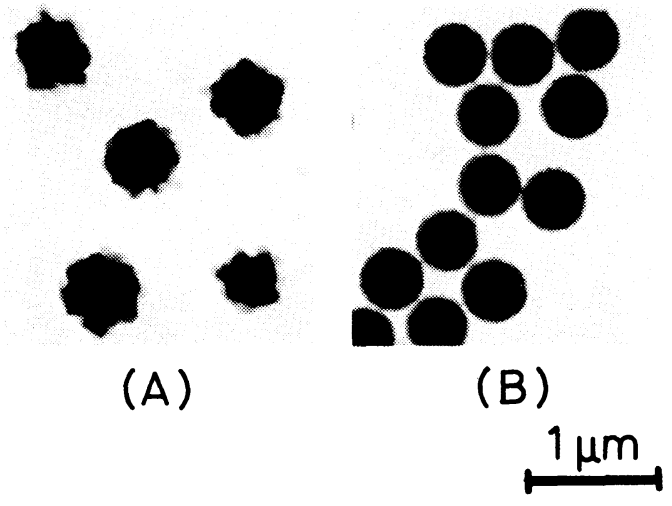

Figure 9. Transmission electron micrographs of StAPr particles prepared under different initiator concentrations at $60^{\circ} \mathrm{C}, f=0.3$. [V 50]: (A) $10.24 \mathrm{mmol} \mathrm{l}^{-1}$ and (B) $2.05 \mathrm{mmol} \mathrm{1}^{-1}$.

mation of spherical particles having a smooth surface.

\section{Effect of Initiator Concentration on Mor- phology}

Particle morphology was controlled by the species and concentrations of initiators and the viscosity of polymerization loci in a poly(MMA) seeded emulsion polymerization of St. $^{21}$ It was emphasized by the authors that the anchoring effect arising from terminal ionic groups is an important factor determining the particle morphology and an increase in the initiator concentration causes the formation of uneven-shaped particles.

$\mathrm{St}-\mathrm{APr}$ copolymerization is regarded as a kind of seeded polymerization and the effect of the initiator concentration on morphology was examined to check whether the above situation comes about or not in our system. The particles obtained at different initiator concentrations are shown in Figure 9. It was confirmed that a decrease in the initiator concentration made the particle surface smoother and the result supported the theory of the anchoring effect.

The inner structure of SAPr particles prepared at a low initiator concentration is shown in Figure 10, which shows that the particles 


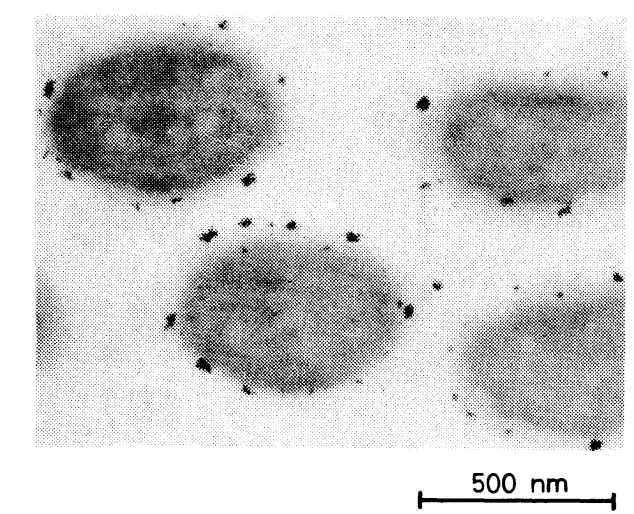

Figure 10. Transmission electron micrograph of $\mathrm{OsO}_{4}$ stained and thinly sectioned $\mathrm{St}-\mathrm{APr}$ particles. Latex preparation: $[\mathrm{V} 50]=2.05 \mathrm{mmol} \mathrm{l}^{-1} ; f=0.3$; temperature $=60^{\circ} \mathrm{C}$. Small spots are considered aggregates of osmic compounds.

contain no appreciable St domains.

\section{Effect of Solvent Addition on Morphology}

Difference in the polymerization timing between two monomers, St and APr, is one of the reasons for phase separation inside the particles. The timing must be variable by the concentration of St dissolved in the aqueous medium and an increase in the concentration of $\mathrm{St}$ is supposed to give more randomly copolymerized chains or particle nuclei in the medium. These products must depress the phase separation. $N, N$-Dimethylformamide (DMF) was selected as a cosolvent which enables St to dissolve in the medium at higher concentration and to polymerize competitively or randomly with APr. Figure 11 shows that the addition of DMF to an aqueous medium caused an increase in the concentration of St dissolved in the medium although that of APr was not affected by DMF addition. The copolymerization of St with APr was carried out in a few aqueous DMF solutions and the shapes of resulting particles are shown in Figure 12. The last micrograph in Figure 6 should be regarded as a control for those in Figure 12. The results in Figure 12 indicate that the addition of more than $10 \%$ DMF was effective

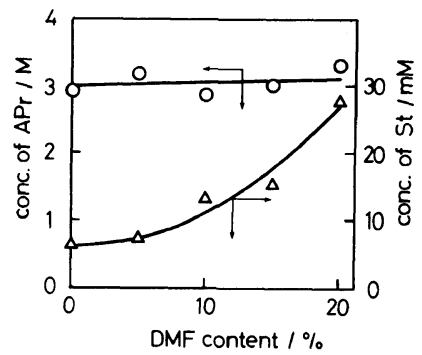

Figure 11. Effects of DMF addition on the concentration of APr and St dissolving in water phase at $60^{\circ} \mathrm{C}$, $f=0.3$.

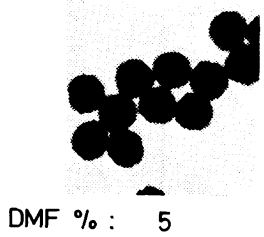

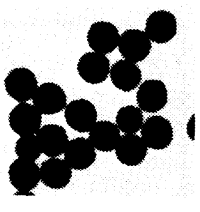

10

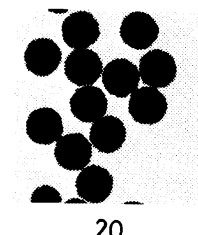

$500 \mathrm{~nm}$
Figure 12. Effect of DMF addition on particle morphology. Latex preparation: $f=0.3$; temperature $=60^{\circ} \mathrm{C}$.

to obtain spherical particles even if the polymerization was carried out with $f=0.3$ at $60^{\circ} \mathrm{C}$.

To examine the mechanism of formation of spherical particles in the presence of DMF, the inner structure of particles prepared in $10 \%$ DMF was observed. A homogeneous inner structure was expected because DMF addition should bring about more take-up of St units into chains in aqueous-phase polymerization and such chains should form nuclei capable of preventing phase separation in the following polymerization. But the results in Figure 13 indicate that this is not the case and the particles have a core-shell structure. This means that the formation of spherical particles by DMF addition did not result from the promoted copolymerization of St with APr in the aqueous phase.

The viscosity in growing particles is one of the factors to decide particle morphology as mentioned previously. Therefore, the dilution effect of DMF in the particles was checked by measuring the degree of swelling of poly(APr) gel in water, St and DMF. The results in Table 


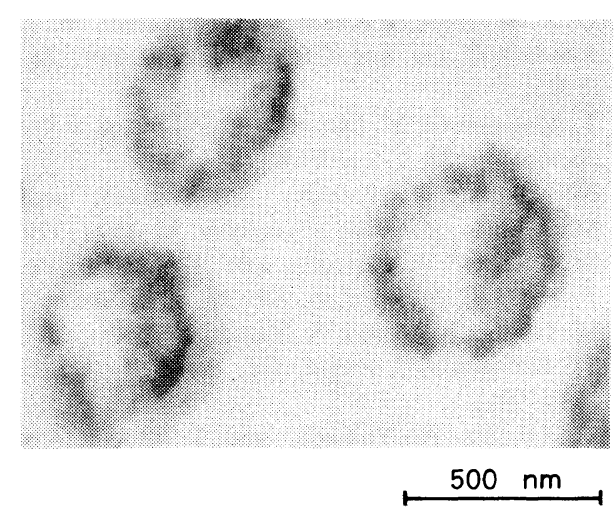

Figure 13. Transmission electron micrograph of $\mathrm{OsO}_{4}$ stained and thinly sectioned $\mathrm{St}-\mathrm{APr}$ particles. Latex preparation: medium $=10 \% \mathrm{DMF} ; f=0.3$; temperature $=60^{\circ} \mathrm{C}$.

Table I. The degree of swelling of poly(APr) gel spheres at $60^{\circ} \mathrm{C}$

\begin{tabular}{lc}
\hline Solvent & $\left(W_{\mathrm{w}} / W_{\mathrm{d}}\right)^{\mathrm{a}}$ \\
\hline $\mathrm{H}_{2} \mathrm{O}$ & 3.1 \\
$\mathrm{DMF}$ & 3.9 \\
$\mathrm{St}$ & 4.2 \\
\hline
\end{tabular}

a $W_{\mathrm{w}}$, weight of swollen gel spheres; $W_{\mathrm{d}}$, weight of dry gel spheres.

Table II. Cloud points of poly(APr) in different solvents

\begin{tabular}{cc}
\hline Solvent & Cloud point $/{ }^{\circ} \mathrm{C}$ \\
\hline $\mathrm{H}_{2} \mathrm{O}$ & 47 \\
$10 \%$ DMF aq & 63 \\
\hline
\end{tabular}

I show that the compatibility of St and DMF with poly(APr) is higher than that of water at $60^{\circ} \mathrm{C}$ and these organic solvents serve to swell poly(APr)-rich growing particles. The extent of affinity of poly(APr) with DMF-containing media is estimated from the cloud point of poly(APr) in the media. Table II shows the cloud points as a function of DMF concentration in the media. It is concluded from these results that DMF addition in the polymerization system causes a looser or more swollen structure of growing particles, gives

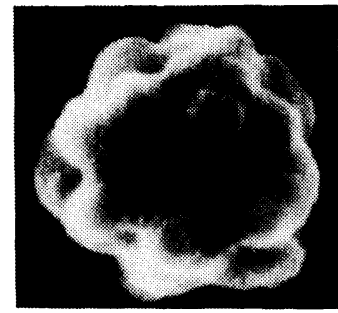

(A)

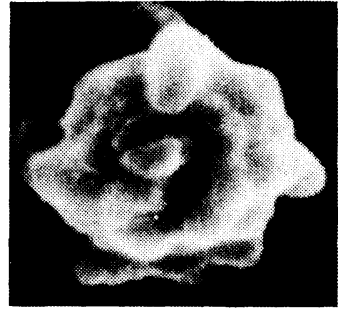

(B)

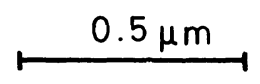

Figure 14. Scanning electron micrographs of St-APr particles dried under different conditions. (A) freezedried and (B) dried at $60^{\circ} \mathrm{C}$. Latex preparation: $f=0.3$; temperature $=60^{\circ} \mathrm{C}$.

high mobility to propagating chains, and finally makes particles having a hydrophobic core and hydropilic shell.

\section{Actual Shape of SAPr Particles in Water}

All the above discussion on particle morphology is based on observations of electron micrographs of dried particles which might be different from those of dispersing particles in water. The actual shape of dispersing particles is hard to observe but an attempt to do so was done by preparing freeze-dried particles. Figure 14 presents the shapes of a freeze-dried particle and a particle dried at $60^{\circ} \mathrm{C}$ in vacuo. The former has fewer projections and is larger than the latter. The results imply that the shape of dried particles might not be a real one but one distorted or shrunken more or less in the course of dehydration. Observation of such distorted or shrunken shapes is valuable in a sense because they can give more information about the inner structure of particles.

\section{CONCLUSIONS}

Morphology of copolymer latex particles of St and acylamide derivatives depended significantly on the polymerization mode and slightly on the polymerization temperature. A high $\mathrm{APr} / \mathrm{St}$ ratio in charge gave unevenlyshaped particles. Such shape was changed to a 
spherical and smooth one by lowering the initiator concentration or adding DMF into the system. These variaions were explained by the mode extent of phase separation in each particle and are funcitions of polymerization timing of the two monomers and the viscosity in the growing particle.

Acknowledgements. The authors gratefully acknowledge the invaluable assistance of $\mathrm{Mr}$. T. Fujiwara, Electron Microscope Laboratory, Keio University, and Mr. N. Kuramitsu, Mitsui Toatsu Chemicals Inc., for the helpful advice on electron microscopy.

\section{REFERENCES}

1. D. I. Lee, "Emulsion Polymers and Emulsion Polymerization," D. R. Bassett and A. E. Hamielec, Ed., ACS Symp. Ser., 165, 405 (1981).

2. D. I. Lee and T. Ishikawa, J. Polym. Sci., Polym. Chem. Ed., 21, 147 (1983).

3. S. C. Misra, C. Pichot, M. S. El-Aasser, and J. W. Vanderhoff, J. Polym. Sci., Polym. Chem. Ed., 21, 2383 (1983).

4. T. I. Min, A. Klein, M. S. El-Aasser, and J. W. Vanderhoff, J. Polym. Sci., Polym. Chem. Ed., 21, 2845 (1983).

5. V. Dimonie, M. S. El-Aasser, A. Klein, and J. W. Vanderhoff, J. Polym. Sci., Polym. Chem. Ed., 22, 2197 (1984).
6. D. R. Stutman, A. Klein, M. S. El-Aasser, and J. W. Vanderhoff, Ind. Eng. Chem. Prod. Res. Dev., 24, 404 (1985).

7. T. Matsumoto, M. Okubo, and S. Shibao, Kobunshi Ronbunshu, 33, 575 (1976).

8. M. Okubo, Y. Katsuta, A. Yamada, and T. Matsumoto, Kobunshi Ronbunshu, 36, 459 (1979).

9. M. Okubo, Y. Katsuta, and T. Matsumoto, $J$. Polym. Sci., Polym. Lett. Ed., 18, 481 (1980).

10. M. Okubo, M. Ando, A. Yamada, Y. Katsuta, and T. Matsumoto, J. Polym. Sci., Polym. Lett. Ed., 19, 143 (1981).

11. M. Okubo, A. Yamada, and T. Matsumoto, J. Polym. Sci., Polym. Chem. Ed., 16, 3219 (1981).

12. R. A. Cox, M. C. Wilkinson, and J. M. Creasey, J. Polym. Sci., Polym. Chem. Ed., 15, 2311 (1977).

13. S. Kamei, M. Okubo, and T. Matsumoto, Preprints, the 3rd Polymer Microspheres Symposium in Japan, 1984, p 53.

14. C. F. Zukoski and D. A. Saville, J. Colloid Interface Sci., 104, 583 (1985).

15. Y. Ohtsuka, H. Kawaguchi, and Y. Sugi, J. Appl. Polym. Sci., 26, 1637 (1981).

16. H. Kawaguchi, Y. Sugi, and Y. Ohtsuka, J. Appl. Polym. Sci., 26, 1649 (1981).

17. H. Kawaguchi, F. Hoshino, and Y. Ohtsuka, Makromol. Chem., Rapid Commun., 7, 109 (1986).

18. G. L. Stahl, R. Walter, and C. W. Smith, J. Am. Chem. Soc., 101, 5383 (1979).

19. F. Hoshino, H. Kawaguchi, and Y. Ohtsuka, Polym. J., 19, 241 (1987).

20. B. Yamada and T. Otsu, Kobunshi Ronbunshu, 37, 351 (1980).

21. I. Cho and K.-W. Lee, J. Appl. Polym. Sci., 30, 1903 (1985). 